\title{
EDITORIAL
}

\section{A new journal in optics and photonics - Light: Science \& Applications}

\author{
Light: Science \& Applications (2012) 1, e3; doi:10.1038/Isa.2012.3; published online 29 March 2012
}

A fter extensive cooperative discussion and planning, the I Changchun Institute of Optics, Fine Mechanics and Physics (CIOMP), Chinese Academy of Sciences and Nature Publishing Group are proud to launch the copublished academic journal, Light: Science \& Applications. I consider it a great honor to be able to write this Editorial at the birth of this journal, and I put my pen to paper here, filled with emotion and enthusiasm.

China is an ancient civilization with a rich recorded history that dates back over 3000 years, but China's involvement in research and development in modern science and technology has come only recently. Indeed, the importation of modern science and technology to China has only occurred within the last 200 years, and this served as the foundation for the subsequent large-scale research and development activities in the 1950s. The establishment of premier academic journals, such as Light: Science \& Applications, will provide a medium for the dissemination and exchange of knowledge and ideas with colleagues around the world, which, in turn, will serve to inspire and direct the future and advancement of modern science.

In recent years, with the strong support and encouragement from Chinese society as a whole, China has experienced rapid progress in science and technology. In 2011, the funding support of R\&D activities reached $1.83 \%$ of GDP, with an average annual growth of more than $20 \%$ since the beginning of the twenty-first century. This funding is undoubtedly the highest among all developing countries in the world, in terms of both a relative index and absolute numbers. The number of people employed in R\&D activities has also increased rapidly, with 2.8 million full-time researchers in 2011, among the highest numbers in the world. The R\&D activities in China annually generate a huge number of scientific papers published in thousands of scientific and technological journals. These facts all demonstrate that China is now in a position of prominence in scientific research and development on the global stage.

However, while China is a prominent country in terms of the scale of research being conducted, it still has to reach its full potential in contributing to novel and innovative research in the fields of modern science and technology. In China, the R\&D activities largely focus on applied research to solve the current problems in engineering and industry. This means that forward-looking, pioneering and original work is less when compared to certain other countries, and this can be difficult to attract the entire science and technology community's attention and support. Nevertheless, China continues to develop at pace, and the desire to reach out to the world and fully participate with colleagues from all countries in all aspects of optics and photonics research has led to the launch of Light: Science \& Applications. This journal will showcase the best international optics and photonics research and, through rigorous but fair peer-review, publish the most outstanding and interesting work possible. Light: Science \& Applications is a fully open access journal-the first Chinese-based, completely open access journal in the physical sciences to be published by Nature Publishing Group. I take this opportunity to express my heartfelt thanks to our partners, academic colleagues, and all the staff for their tireless efforts and support to establish this journal.

The partnership between Nature Publishing Group and CIOMP utilizes the core strengths of both organizations. NPG is clearly a leader in the scientific publishing world. CIOMP, one of the new China's early national research institutions founded in 1952, has, over the course of 60 years and under the leadership of Academician Daheng Wang, undertaken a series of pioneering works across many fields. These include applied optics, precision mechanics, lasers and optoelectronics, and CIOMP has been hailed as "the Cradle of Optics in China". In the different phases of China's development, CIOMP has always played a crucial role in meeting the needs of the nation's development, social environment and research requirements.

My role at the Ministry of Science and Technology, with one of the divisions responsible for international scientific and technological exchange and cooperation, has convinced me that the exchange of scientific knowledge is most successful when it is freely exchanged and available to all, regardless of geography. The general consensus is that information on scientific and technological activities should be a common human practice. The processes and the outcomes of research should be widely disseminated to the world, so that any person who wants to access such information would be able to achieve in a timely and efficient manner. The advancements should not, and cannot, be confined to a few intellects in a few areas or in a few countries. A popular Chinese classical poem goes "Where once the swallows knew the mansions of the great/They now to humbler homes would fly to nest and mate", which sums up nicely for me the present state of science and technology. In fact, more countries and more people learning to master the use of technology is the common goal of human society. I believe that Light: Science \& Applications can play a very important role in achieving this goal.

I wish to thank current and future authors and readers for their support, and wish Light: Science \& Applications all success!

Jianlin Cao

Editor-in-Chief, Light: Science \& Applications Vice Minister of Ministry of Science and Technology, Professor of Film Optics, State Key Laboratory of Applied Optics, CIOMP, Dong Nanhu Road 3888, Changchun, China

This work is licensed under a Creative Commons Attribution-NonCommercial-NoDerivative Works 3.0 Unported License. To view a copy of this license, visit http:// creativecommons.org/licenses/by-nc-nd/3.0 